\title{
The Brera Multi-scale Wavelet ROSAT HRI source catalogue ${ }^{\star}$
}

\author{
M. R. Panzera ${ }^{1}$, S. Campana ${ }^{1}$, S. Covino ${ }^{1}$, D. Lazzati ${ }^{1,2}$, R. P. Mignani ${ }^{3}$, A. Moretti ${ }^{1}$, and G. Tagliaferri ${ }^{1}$ \\ 1 INAF - Osservatorio Astronomico di Brera, via E. Bianchi 46, 23807 Merate (LC), Italy \\ 2 Institute of Astronomy, University of Cambridge, Madingley Road, CB3 0HA Cambridge, UK \\ ${ }^{3}$ European Southern Observatory, Karl Schwarzschild Strasse 2, 85740 Garching bei München, Germany
}

Received 2 August 2002 / Accepted 24 October 2002

\begin{abstract}
We present the Brera Multi-scale Wavelet ROSAT HRI source catalogue (BMW-HRI) derived from all ROSAT HRI pointed observations with exposure times longer than $100 \mathrm{~s}$ available in the ROSAT public archives. The data were analyzed automatically using a wavelet detection algorithm suited to the detection and characterization of both point-like and extended sources. This algorithm is able to detect and disentangle sources in very crowded fields and/or in the presence of extended or bright sources. Images have been also visually inspected after the analysis to ensure verification. The final catalogue, derived from 4303 observations, consists of 29089 sources detected with a detection probability of $\geq 4.2 \sigma$. For each source, the primary catalogue entries provide name, position, count rate, flux and extension along with the relative errors. In addition, results of cross-correlations with existing catalogues at different wavelengths (FIRST, IRAS, 2MASS and GSC2) are also reported. Some information is available on the web via the DIANA Interface. As an external check, we compared our catalogue with the previously available ROSHRICAT catalogue (both in its short and long versions) and we were able to recover, for the short version, $\sim 90 \%$ of the entries. We computed the sky coverage of the entire HRI data set by means of simulations. The complete BMW-HRI catalogue provides a sky coverage of $732 \mathrm{deg}^{2}$ down to a limiting flux of $\sim 10^{-12} \mathrm{erg} \mathrm{s}^{-1} \mathrm{~cm}^{-2}$ and of $10 \mathrm{deg}^{2}$ down to $\sim 10^{-14} \mathrm{erg} \mathrm{s}^{-1} \mathrm{~cm}^{-2}$. We were able to compute the cosmological $\log (N)-\log (S)$ distribution down to a flux of $\simeq 1.2 \times 10^{-14} \mathrm{erg} \mathrm{s}^{-1} \mathrm{~cm}^{-2}$.
\end{abstract}

Key words. catalogs - X-rays: general

\section{Introduction}

Since the early phases of X-ray astronomy, source catalogues have been regularly compiled either from systematic sky surveys or from the collection of serendipitous observations, especially when imaging X-ray telescopes became available (e.g. Einstein EMSS, Gioia et al. 1990; EXOSAT HGSC, Giommi et al. 1991; ROSAT WGA, White et al. 1994; ROSAT SRC, Zimmermann 1994; ASCA SIS, Gotthelf \& White 1997). Depending on the telescope field of view and mission lifetime, these surveys covered $1-10 \%$ of the sky and allowed investigators to pursue statistical studies on virtually all classes of X-ray emitting sources.

Observations with the ROSAT PSPC produced a number of catalogues; besides the RASS-BSC (Voges et al. 1999), derived from the ROSAT All Sky Survey, there are the WGA (White et al. 1994) and the ROSPSPC (ROSAT Team 2001), derived from the collection of all PSPC pointed observations. On the contrary the large database of ROSAT High Resolution

Send offprint requests to: M. R. Panzera,

e-mail: panzera@merate.mi . astro.it

* The catalogue is only available in electronic form at the CDS via anonymous ftp to cdsarc.u-strasbg.fr $(130.79 .128 .5)$ or via http://cdsweb.u-strasbg.fr/cgi-bin/qcat?J/A+A/399/351
Imager (HRI) observations has been only marginally exploited. Rather recently (August 2001), a full catalogue based on the Standard Analysis Software System (SASS) has been released (ROSHRICAT, ROSAT Team 2001). This catalogue contains arcsecond positions and count rates for automatically and visually inspected sources, including 13452 high confidence $(S / N>4)$ detections, from 5393 public ROSAT HRI observations covering $1.94 \%$ of the sky. However, the SASS suffers from some limitations (e.g. 331 "obvious" sources were added manually since they were not detected by the SASS), not least the fact that only bright sources can be securely detected.

The HRI on board the ROSAT satellite is a microchannel plate detector with an octagon shaped field of view (with $\sim 19^{\prime}$ radius) that reveals single $\mathrm{X}$-ray photons providing information on their positions and arrival times. The HRI Point Spread Function (PSF) as measured on-axis is of about 5 arcsec $F W H M$, i.e. a factor of $\sim 4$ better than the one of ROSAT-PSPC (and just a factor of $\sim 5$ worse than Chandra). The sharp core of the HRI PSF allows us to detect and disentangle sources in very crowded fields and to detect extended emission on a small angular size. On the other hand, the HRI has a very crude spectral resolution in the $0.1-2.4 \mathrm{keV}$ energy band (for more details see Prestwich et al. 1996), it is less efficient than the PSPC 
(a factor of 3 to 8 for a plausible range of incident spectra) and it has a higher instrumental background. The ROSAT satellite, its X-ray telescope, and the HRI detector have been described in detail by Pfeffermann et al. (1986), Zombeck et al. (1990), Trümper et al. (1991) and David et al. (1998).

For these reasons, we decided to reanalyze the entire HRI data set with a dedicated source detection algorithm based on the wavelet transform (Lazzati et al. 1999; Campana et al. 1999). The outcome is a new source catalogue named the Brera Multi-scale Wavelet HRI (BMW-HRI). Here we present the results of our analysis. In Sect. 2 we summarize the main characteristics of the detection algorithm. In Sect. 3 we discuss the selection of the ROSAT HRI fields used and we describe the catalogue. In Sect. 4 we present the cosmological $\log (N)-\log (S)$ distribution computed from our catalogue. In Sect. 5 we make a comparison with the ROSHRICAT catalogues. In Sect. 6 we describe the cross-correlations between the BMW-HRI and other catalogues at various wavelengths. Conclusions and catalogue accessibility are reported in Sect. 7.

\section{Wavelet detection algorithm}

The analysis and source detection of HRI images, together with the simulations carried out to test the detection pipeline, are extensively described in Campana et al. (1999) and Lazzati et al. (1999). In this case we decided to run the detection algorithm with a single significance threshold for the sources $(\sim 4.2 \sigma)$ corresponding to a contamination of 0.4 spurious sources per field. We remark here that one of the most interesting features of the wavelet analysis is the possibility of characterizing the source extension (see Sect. 3.2 and Campana et al. 1999). The data were retrieved from the MPE and GSFC public ROSAT databases. Our data processing pipeline analyzes the FITS event files, as produced through the SASS procedure, and the ancillary files that include orbit and spacecraft pointing and other engineering or housekeeping information. From the analysis of each observation we derived a catalogue of sources with position, count rate, extension, along with the relative errors, as well as ancillary information about the observation itself and source fitting. The count-to-flux conversion factor was determined assuming as reference a Crab spectrum (power law with photon index 2.0). A conversion factor was computed both for a low column density $\left(5 \times 10^{19} \mathrm{~cm}^{-2}\right)$ and for the full galactic value. We also applied the corrections due to the vignetting, PSF modelling (we considered a Gaussian fit), plate scale (the nominal pixel size was $0.5^{\prime \prime}$ reduced to $0.4986^{\prime \prime}$ after detailed observations on the Lockman hole field, see Hasinger et al. 1998) and PSF asymmetry at large off-axis angles (see Campana et al. 1999).

\section{The BMW-HRI catalogue}

\subsection{Selection of the ROSAT HRI fields}

The starting point for the BMW-HRI catalogue production was the sample of 4507 ROSAT HRI fields published up to December 2001 with exposure time longer than 100 s. We did not include in our selection the 341 fields pointed on

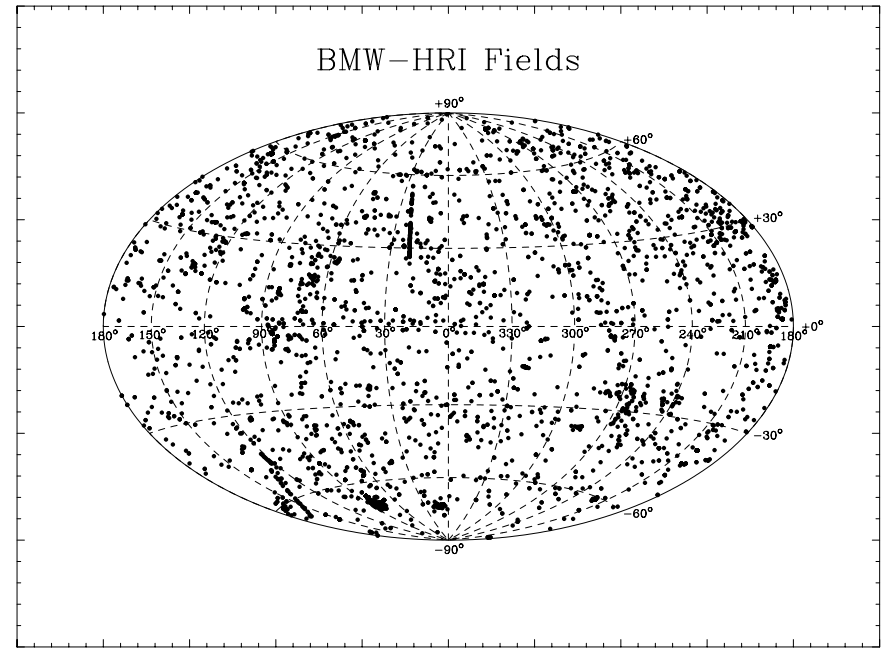

Fig. 1. Aitoff projection in galactic coordinates for the whole set of fields used to construct the BMW-HRI catalogue (4303 observations).

supernova remnants (fields with identification number, ROR, starting with 5) due to the large number of bright overlapping spots in which the remnant is split. This despite the fact that the central source of the (e.g.) Cas A remnant is clearly detected as a point source (Pavlov et al. 2000). We also did not consider the 598 calibration observations (fields with ROR starting with 1). All the 4507 fields were analyzed selfconsistently with the detection algorithm described in Sect. 2. Of the 4507 fields analyzed, $204(\sim 5 \%)$ were discarded due to problems in the construction of the exposure map, or in the reconstruction of the aspect attitude, or due to problems during the analysis. In particular, several fields affected by the spacecraft wobbling during the exposures were discarded because bright sources appeared artificially elongated and where therefore split into at least two sources by the detection algorithm. Moreover, very confused fields like, e.g., the ones targetted on Eta Carinae, were discarded. Finally, a few fields pointed on planets and comets were discarded due to their proper motion which induces multiple detections across the images. Therefore, we ended up with 4303 fields. As our detection threshold corresponds to 0.4 spurious sources per field, we expect $\sim 1721$ spurious sources over all the fields. In 146 fields no sources were detected, due to the too short exposure time. Figure 1 shows the Aitoff projection in galactic coordinates for the 4303 observations.

The distribution of the exposure times can be roughly described by a double Gaussian distribution with $\sim 3$ and $\sim 25 \mathrm{ks}$ as centroid peaks (see Fig. 2). In Fig. 3 we plot the distribution of the galactic hydrogen column density after Dickey \& Lockman (1990).

\subsection{Catalogue characteristics}

The BMW-HRI catalogue consists of 29089 sources down to a limiting ROSAT HRI count-rate of $\sim 10^{-4}$ count $\mathrm{s}^{-1}$ covering $\sim 1.8 \%$ of the sky. A detailed description of all the BMW-HRI parameters together with the ones derived from the crosscorrelations (see Sect. 6) can be found in the Appendix A 


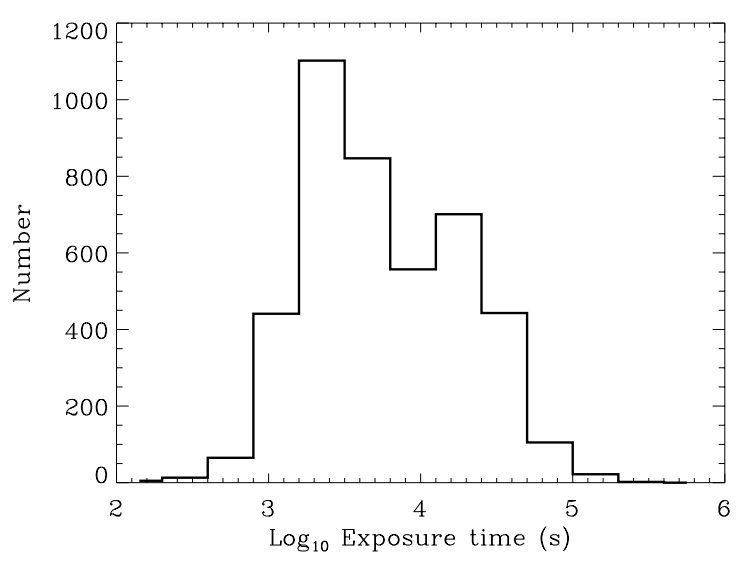

Fig. 2. Distribution of the exposure times for the 4303 ROSAT-HRI observations considered.

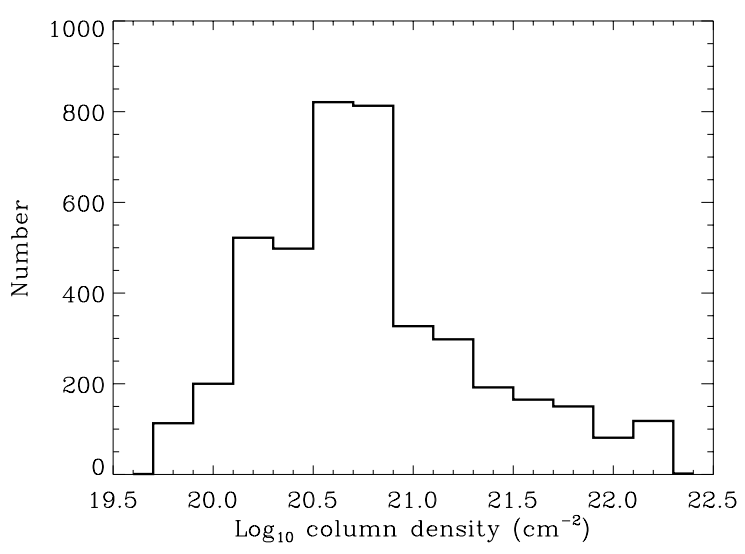

Fig. 3. Distribution of the galactic column density after Dickey \& Lockman (1990) for the 4303 ROSAT-HRI observations considered.

(Tables A.1-A.5). The catalogue lists all the sources detected in all the 4303 fields analyzed, i.e. we did not associate sources detected in different observations of the same area of the sky. An estimate of the number of independent sources in the catalogue can be obtained compressing the number of sources using an error on the source position of $10^{\prime \prime}$ (a conservative limit dictated by the uncertainties connected with the boresight correction). We found 20453 independent objects.

In Fig. 4 we plot the distribution of source off-axis angles showing the typical trend due to the increase of the collecting area together with the decreasing of the sensitivity with offaxis. Clearly, the peak at zero off-axis is due to pointed sources.

One of the most important characteristics of wavelet algorithms is the ability to determine the source extension, i.e. the scale of the wavelet transform where the $S / N$ is maximized after the application of the fitting refinement procedure (see Lazzati et al. 1999), and, if a criterion is given, also to disentangle point and extended sources (see Campana et al. 1999). To assess the source extension criterion we considered all sources detected in the observations that have a star(s) as a target (ROR number beginning with 2) and that were available in the public archives in a preliminary phase of our catalogue: 6013 sources in 756 HRI fields. The distribution of the source extension as a function of the source off-axis angle has been divided into bins of $1 \mathrm{arcmin}$ each. To each bin we then applied a $\sigma$-clipping

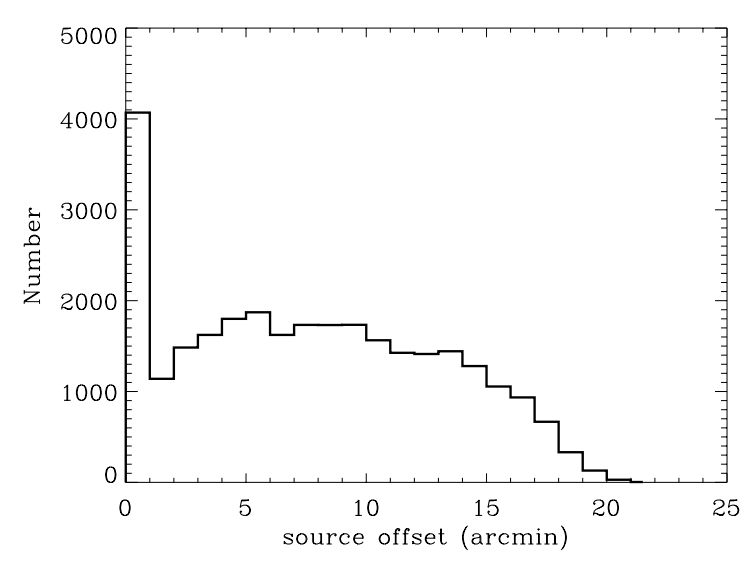

Fig. 4. Distribution of the off-axis angles for the 29089 BMW-HRI sources. The plot shows the typical trend due to: targets plus increasing of the collecting area together with the decreasing of the sensitivity with the off-axis.

algorithm to discard iteratively truly extended sources and to derive the mean value of the source extension in the bin for pointed sources. We then determined the $3 \sigma$ dispersion on the mean for each bin. The mean value plus the $3 \sigma$ dispersion provides the threshold for the source extension. We conservatively classify a source as extended if it lies more than $2 \sigma$ from this limit (i.e. if the source extension error bar lies more than twice from the $3 \sigma$ limit described above). Combining this threshold with the $3 \sigma$ on the intrinsic dispersion, we obtain a $\sim 4.5 \sigma$ confidence level for the extension classification (see also Rosati et al. 1995). In Fig. 5 we plot the distribution of the source extension versus off-axis angle for the 29089 BMW-HRI sources (small dots). The solid line in Fig. 5 represents the mean value of source extension for pointed sources as described above, while the dashed line is the $3 \sigma$ dispersion on this mean. Open squares in Fig. 5 represent sources we classified as extended, i.e. with a confidence level for the extension classification of $\sim 4.5 \sigma$.

We end up with 2717 extended sources (open squares in Fig. 5) containing supernova remnants, galaxies, cluster of galaxies etc. (as well as blending of nearby sources). The distributions of source extensions for the truly extended sources, the full sample and only the high-galactic latitude $\left(|b|>20^{\circ}\right)$ one (2139 sources), are reported in Fig. 6. We assumed that all the high-galactic latitude extended sources are extragalactic in nature. In the plot we also report the on-axis angular resolution of the ROSAT PSPC. X-ray extensions are calculated subtracting in quadrature the relative PSF extension at a given off-axis angle (solid line in Fig. 5). The extended sources were used to select a list of candidate X-ray selected cluster of galaxies that we then studied with optical follow-up (Moretti et al. 2002 in preparation; Guzzo et al. 2002 in preparation).

As the sensitivity of the HRI instrument is not uniform over the entire field of view, for a given limiting flux the surveyed area does not coincide with the detector one but it is generally smaller. We calculated the sky coverage of the entire survey as a function of the flux (calculated with the full column density) by means of simulations. To this aim we performed extensive Montecarlo tests using the detection procedures on 


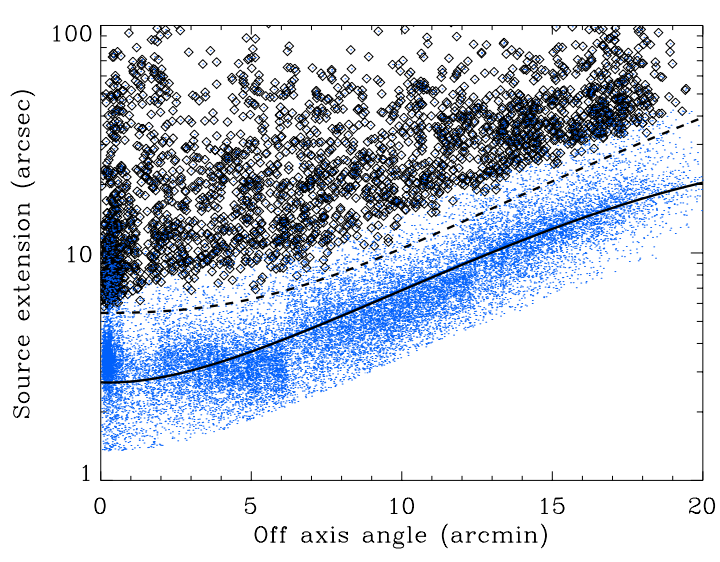

Fig. 5. Distribution of BMW-HRI source extensions. The solid line represents the HRI PSF, whereas the dashed line the $3 \sigma$ limit for point sources as described in the text. Small dots represent the extension for all sources. Open squares denote truly $(\sim 4.5 \sigma)$ extended sources i.e. sources that lies more than $2 \sigma$ from the dashed line (2717 sources).

the simulated data for 12 observations with different exposure times (i.e. $1680 \mathrm{~s}, 2656 \mathrm{~s}, 4976 \mathrm{~s}, 10000 \mathrm{~s}, 16080 \mathrm{~s}$, 20664 s, 27488 s, 42672 s, 52880 s, 80288 s, 107712 s and $200272 \mathrm{~s}$ ). For each field of the survey we derived a completeness function that gives the detection probability as a function of flux and position of point-like sources. Because of the worsening of the PSF, within the same field, the detection probability of a given flux decreases for increasing off-axis angle. Therefore, for each observation the sky coverage is the integral of the completeness function over the field of view. Moreover, each observation has different completeness function depending on the exposure time and on the column density values. The sky coverage of the whole survey is the sum of the contributions of each single field. Some of the fields used to build the BMW-HRI catalogue cover the same area of the sky: in this case in the calculation of the total sky coverage we considered only the contribution from the longest observation performed over that area. All the procedures of data simulations and the features of the Montecarlo tests are fully described in Moretti et al. (2002, in preparation). The complete sky coverage for point-like sources is shown in Fig. 7 (for extended sources see Moretti et al. 2002 in preparation). The maximum area of the survey is $\sim 732 \mathrm{deg}^{2}$ and corresponds to fluxes above $\sim 10^{-12} \mathrm{erg} \mathrm{s}^{-1} \mathrm{~cm}^{-2}$. At $10^{-13} \mathrm{erg} \mathrm{s}^{-1} \mathrm{~cm}^{-2}$ the surveyed area is $\sim 314 \mathrm{deg}^{2}$ and $\sim 10 \mathrm{deg}^{2}$ at $10^{-14} \mathrm{erg} \mathrm{s}^{-1} \mathrm{~cm}^{-2}$.

\section{4. $\log (N)-\log (S)$}

In order to compute the integral flux distribution $(\log (N)-\log (S))$ of cosmological sources we used a subsample of the catalogue. Firstly, we selected all high galactic latitude fields $\left(|b|>30^{\circ}\right)$ observed for more than $5 \mathrm{ks}$. Then, we filtered out those containing extended sources (like NGC objects and galaxies clusters), Magellanic Clouds targets or crowded areas (e.g. M 31). Finally, from the remaining fields, we chose sources with off-axis angles between 3 and 15 arcmin. To summarize, we restricted to 501 fields $\left(\sim 90 \mathrm{deg}^{2}\right)$ and 3161 sources. The survey is inhomogeneous

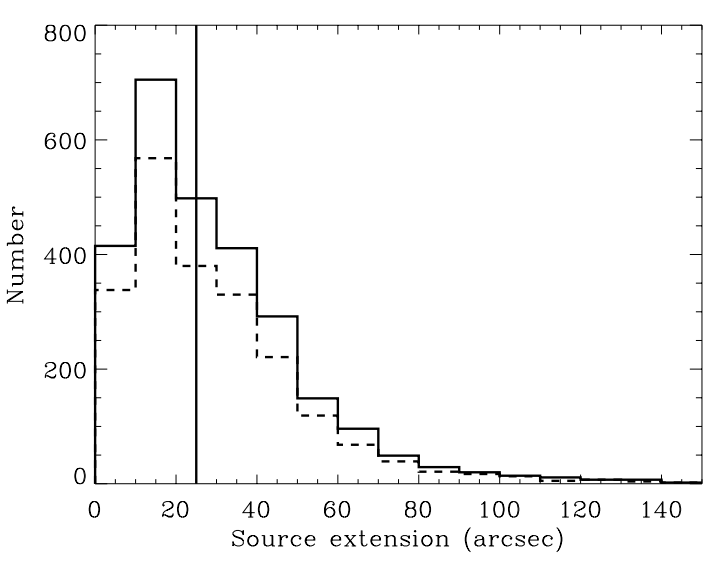

Fig. 6. Distribution of BMW-HRI source extensions for the 2717 extended sources (solid line). The dashed line represents the distribution of the high-galactic latitude $\left(|b|>20^{\circ}\right)$ extended sources (2139 objects), whereas the vertical solid line the on-axis angular resolution of the ROSAT PSPC. The X-ray extensions are calculated subtracting in quadrature the intrinsic PSF width.

(because of the worsening of the PSF with the off-axis) thus in the computation of the flux distribution different sources have different weights. The weight is defined as the inverse of the area in which the source has a non-zero probability of being detected. Our results are compared in Fig. 8 with those of the ROSAT Deep Survey (Hasinger et al. 1998). We derive our distribution down to $\simeq 1.2 \times 10^{-14} \mathrm{erg} \mathrm{s}^{-1} \mathrm{~cm}^{-2}$ where the surveyed area corresponds to $8 \%$ of the total $\left(\simeq 8 \mathrm{deg}^{2}\right)$. The ROSAT Deep Survey extends to fainter fluxes and it is well fitted by a broken power law, with the break at $\sim 2 \times 10^{-14} \mathrm{erg} \mathrm{s}^{-1} \mathrm{~cm}^{-2}$ (Hasinger et al. 1998). The BMW-HRI distribution is very similar both in steepness and in normalization to the ROSAT Deep Survey, but extends to brighter fluxes, a factor of 2 after the break point. In order to compare the two distributions, we exclude from the BMW-HRI distribution the fainter fluxes $\left(<7 \times 10^{-14} \mathrm{erg} \mathrm{s}^{-1} \mathrm{~cm}^{-2}\right.$; this is why we cannot constrain the power law below the break with such few points) and we compared the BMW-HRI distribution with the bright part of the ROSAT Deep Survey. By means of a maximum likelihood minimization fit we find that, assuming a single power law for the differential distribution, the best fit for the exponential is given by $\alpha=-2.75 \pm 0.11$ with a normalization of $229.8_{-60}^{+69}$ (in units of $10^{-14}$ ). This value is in very good agreement with the bright part of the ROSAT Deep Survey flux distribution $(\alpha=-2.72$ and a normalization of 238.1; Hasinger et al. 1998).

\section{Comparison with the ROSHRICAT catalogues}

We compared the BMW-HRI with the ROSAT source catalogue of pointed observations with the High Resolution Imager (ROSHRICAT/1RXH, ROSAT Team 2001). This catalogue, derived by reprocessing the public HRI dataset (a total of 5393 pointings covering $1.94 \%$ of the sky) through the SASS, provides arcsecond positions and count rates for 131902 sources. This version includes detections which were classified as false after a visual inspection ("f $\mathrm{f}$ " detections), multiple detections of 


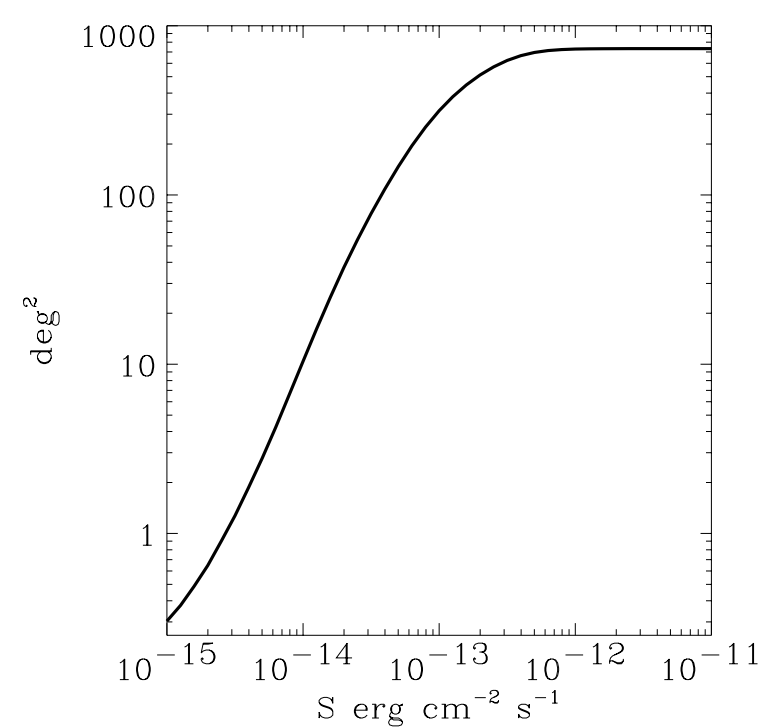

Fig. 7. Sky coverage for point-like sources of the entire survey as a function of the flux (full column density): the maximum area of the survey is $\sim 732 \mathrm{deg}^{2}$ and corresponds fluxes above $10^{-12} \mathrm{erg} \mathrm{s}^{-1} \mathrm{~cm}^{-2}$. At $10^{-13} \mathrm{erg} \mathrm{s}^{-1} \mathrm{~cm}^{-2}$ the surveyed area is $\sim 314 \mathrm{deg}^{2}$ and $\sim 10 \mathrm{deg}^{2}$ at $10^{-14} \mathrm{erg} \mathrm{s}^{-1} \mathrm{~cm}^{-2}$.

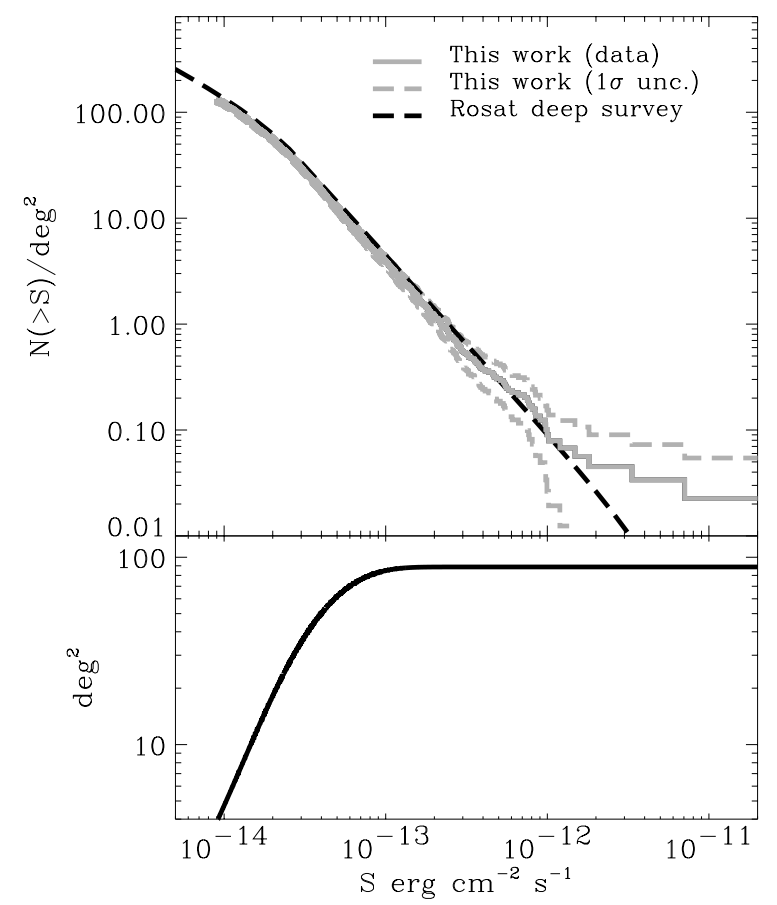

Fig. 8. Upper panel: comparison between the BMW-HRI $\log (N)-\log (S)$ derived for the cosmological sources (bold gray solid line) and the ROSAT Deep Survey distribution (dashed line; Hasinger et al. 1998). The two thin gray solid lines represents our $1 \sigma$ uncertainties. The comparison is made in the bright part of ROSAT Deep Survey (see text); lower panel: sky coverage of the 501 selected fields as a function of the flux (see text).

the same source within the same observation ("u" detections) and 331 obvious sources which were not detected by the SASS and added manually (see Appendix A.2). After removing " $u$ " and "f" detections, 56401 entries are left (ROSHRICAT long version). Additionally, applying a $S / N>4$ yields to 13452 high confidence detections (ROSHRICAT short version).

We compared the BMW-HRI catalogue with the ROSHRICAT, both in its short and its long version (hereafter ROSHRICAT-short and ROSHRICAT-long, respectively) by cross-correlating the entries in the two catalogues. The results of these cross-correlations are summarized in this section while a detailed description of specific checks is given in Appendix A. For consistency, we applied to the ROSHRICAT catalogues the same selection criteria applied in the compilations of the BMW-HRI (see Sect. 3). Moreover, we filtered out from the ROSATHRICAT catalogues the 331 entries which were not detected by the SASS (no flux information), plus some detections with a wrong declination (4 sources for the short version and 64 sources for the long version). We remain with 10708 and 43252 entries for the ROSHRICAT-short and long respectively. To compute the cross-correlation radius we first calculated the positional error corresponding to the $95 \%$ of the sources both for the BMW-HRI and for the ROSHRICAT catalogues. By adding in quadrature the two errors we obtain radii of 8 and 12 arcsec, to be used for the cross-correlations with the ROSATHRICAT short and long, respectively. As the number of the ROSHRICAT sources that cross-correlate with each BMW-HRI sources can be, in several cases, more than one, we decided to choose the nearest ROSHRICAT source (minimum distance approach).

\subsection{Comparison with the ROSHRICAT short version}

We first cross-correlated the BMW-HRI (29089 entries) with the ROSATHRICAT-short (10708 entries) using a crosscorrelation radius of 8 arcsec, as determined above, and finding 12442 associations. This means that a single ROSHRICATshort source matches more than one BMW-HRI object. We remember that, for our catalogue, we did not associate objects detected in different observations of the same area of the sky. The number of ROSHRICAT single sources in the crosscorrelation is 9670 . By shifting the coordinates of 3 arcmin we found that the probability of mismatch is of the order of $\sim 0.6 \%$ (that is 73 mismatches). Comparing the ROSHRICAT-short count rates with the BMW-HRI ones we found that a fraction of $87 \%$ has count rates equal within a factor of 2 . 1038 ROSATHRICAT-short sources are found without a correspondence in the BMW-HRI source list. In order to check only firmly detected sources we considered objects with $S / N \geq 5$ (378 entries). We found that: $\sim 62 \%$ have a BMW-HRI association within 30 arcsec; $\sim 32 \%$ are spurious sources while $\sim 6 \%$ are of ambiguous interpretation and in some cases could be true sources lost by our algorithm (a more extensive discussion is given in Appendix A.1).

The number of BMW-HRI sources without a counterpart in the ROSHRICAT short version is 16647 . We discuss the sources lost in the ROSHRICAT catalogues in Sect. 5.2 since a source lost in the short version can have a counterpart in the long version. 


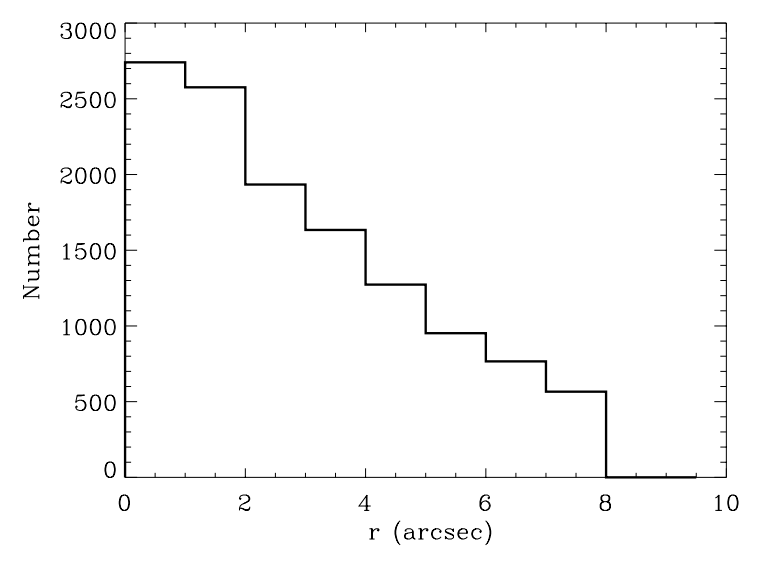

Fig. 9. ROSHRICAT - BMW-HRI: distribution of the angular separation $(r$ ) between ROSHRICAT-short (see text) and BMW-HRI positions for the 12442 cross-correlated objects.

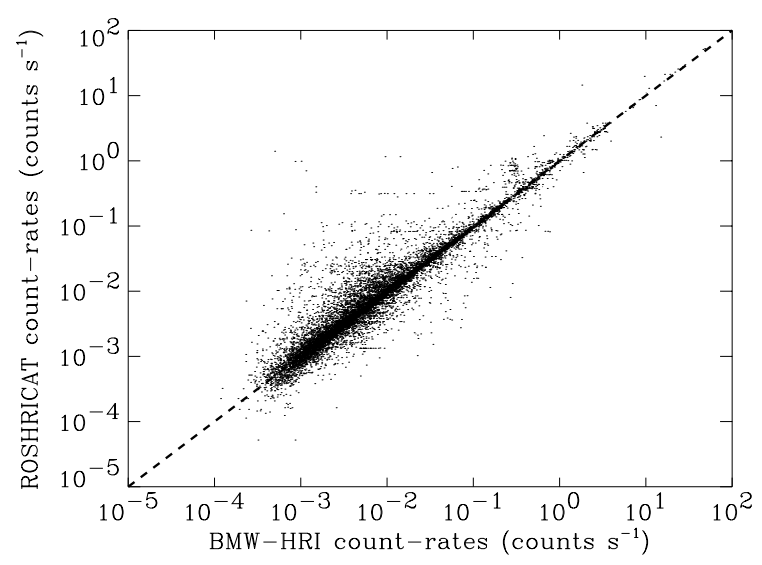

Fig. 10. ROSHRICAT - BMW-HRI: log-log plot of the ROSHRICATshort (see text) count-rates versus BMW-HRI count-rates for the 12442 cross-correlated objects.

In Fig. 9 we show the distributions of the angular separation ( $r$ in arcsec) between ROSHRICAT counterpart position and BMW-HRI position for the 12442 cross-correlated objects.

The BMW-HRI source count-rates for the common entries are plotted (in logarithmic scale) in Fig. 10 vs. the corresponding values from the ROSHRICAT-short.

\subsection{Comparison with the ROSHRICAT long version}

The cross-correlation (radius of 12 arcsec) between BMW-HRI and ROSHRICAT-long (43252 objects) produced 21982 entries. The probability of misidentification is of the order of 3\% (that is 595 mismatches). The number of ROSHRICAT single sources in the cross-correlation is 21120 . Thus 22132 ROSHRICAT sources are not present in the BMW-HRI catalogue. We note that the great majority of them (21924) have $S / N$ lower than 5 .

There are 7107 BMW-HRI sources without a counterpart in the ROSHRICAT-long. Using a cross-correlation radius of 18 arcsec (i.e. corresponding to $3 \sigma$ of the combined average positional error of the two catalogues) the number of unmatched sources reduces to 5870. Since the number of spurious detections expected in the BMW-HRI is $\sim 1721$, we expect that $\sim 30 \%$ of the 5870 sources to be background fluctuations. In order to investigate in more details these 7107 sources, we filtered out sources detected in fields with extended emissions or too crowded on which the SASS algorithm may had problems. Moreover, we decided not to investigate sources with $S / N<5$ as they are near the detection threshold. The analysis of a sub-sample of these sources with $S / N \geq 5$ (1170 objects) shows that: $\sim 49 \%$ are true sources without a counterpart in the ROSHRICAT catalogue; $\sim 36 \%$ have a ROSHRICAT association with angular separation $\geq 18$ arcsec while $\sim 15 \%$ are probably spurious sources (see Appendix A.3). For clarity in Table 1 we put the results of the cross-correlations with the short and long version of the ROSHRICAT respectively.

\section{Cross-correlations with existing data-bases}

Since the sharp core of the ROSAT HRI PSF allows for a more precise determination of the position of an X-ray source, cross-correlations with catalogues at other wavelengths are less affected by spurious matches. This makes the search for counterparts much easier. We cross-correlated the BMW-HRI catalogue with the largest catalogues available at other wavelengths, from radio to optical. For cross-correlation with catalogues at other wavelengths we used (unless otherwise stated) a search radius of $10 \mathrm{arcsec}$. This value comes from the major source of uncertainty in the reconstruction of BMW-HRI source positions, which is the uncertainty in the aspect solution of the ROSAT telescope (i.e. the boresight uncertainty).

\subsection{The FIRST Survey catalogue}

FIRST - Faint Images of the Radio Sky at Twenty-cm - covers 10000 square degrees of the North Galactic Cap. The sensitivity of the survey is of $\sim 1 \mathrm{mJy}$ with an angular resolution of $\sim 5$ arcsec (see Becker et al. 1995). A catalogue containing $\sim 770000$ sources and covering $\sim 8500$ square degrees has been constructed (White et al. 1997). The combined sensitivity and positional accuracy of the FIRST catalogue are unprecedented compared with any previous wide-area radio catalogue. FIRST source locations have an accuracy that matches or exceeds those of all currently available radio catalogues. The cross-correlation with the BMW-HRI found 1019 entries with a misidentification probability of the order of $2 \%$ (that is 18 mismatches). Our cross-correlation is similar to the one made using FIRST and the catalogue of X-ray sources WGACAT from ROSAT PSPC observations (White et al. 1994; see White et al. 1997) and represents one of the largest lists of X-ray/radio coincidences available to date. In Fig. 11 we report the distributions of the angular separation ( $r$ in arcsec) between the radio and the X-ray position for the 1019 matched objects.

In Fig. 12 we plot the integrated flux densities measured in mJy versus X-ray flux (full column density) for the 1019 crosscorrelated sources. The integrated flux is derived by fitting an elliptical Gaussian model to all FIRST sources.

As in the FIRST catalogue we have information about the source extension (the major axis, i.e. the FWHM in arcsec derived from the elliptical Gaussian model for the source) we 
Table 1. BMW-HRI cross-correlation with ROSHRICAT catalogues.

\begin{tabular}{llllll}
\hline \hline $\begin{array}{l}\text { BMW-HRI } \\
\text { entries }\end{array}$ & $\begin{array}{l}\text { ROSHRICAT-short }_{\text {entries }^{a}} \\
29089\end{array}$ & $\begin{array}{l}\text { Radius }^{b} \\
(\operatorname{arcsec})\end{array}$ & $\begin{array}{l}\text { BMW-HRI } \\
\text { cross-correlations }\end{array}$ & $\begin{array}{l}\text { ROSHRICAT-short } \\
\text { cross-correlations }\end{array}$ & Mismatches \\
\hline \hline BMW-HRI & $\begin{array}{l}\text { ROSHRICAT-long }_{\text {entries }} \\
\text { entries }^{a}\end{array}$ & $\begin{array}{l}\text { Radius }^{b} \\
(\operatorname{arcsec})\end{array}$ & $\begin{array}{l}\text { BMW-HRI } \\
\text { cross-correlations }\end{array}$ & $\begin{array}{l}\text { ROSHRICAT-long } \\
\text { cross-correlations }\end{array}$ & Mismatches \\
\hline 29089 & 43252 & 12 & 21982 & 21120 & 595 \\
\hline
\end{tabular}

${ }^{a}$ All the entries of the ROSHRICAT short/long version except: sources corresponding to fields with ROR number 1 and 5; sources corresponding to all the fields we rejected after or during the analysis; the 331 sources not detected by the SASS and the 4/64 sources with a wrong declination (see Sect. 5.1 for more details).

${ }^{b}$ The radius corresponds to $2 \sigma$ on the source positional errors of the two catalogues.

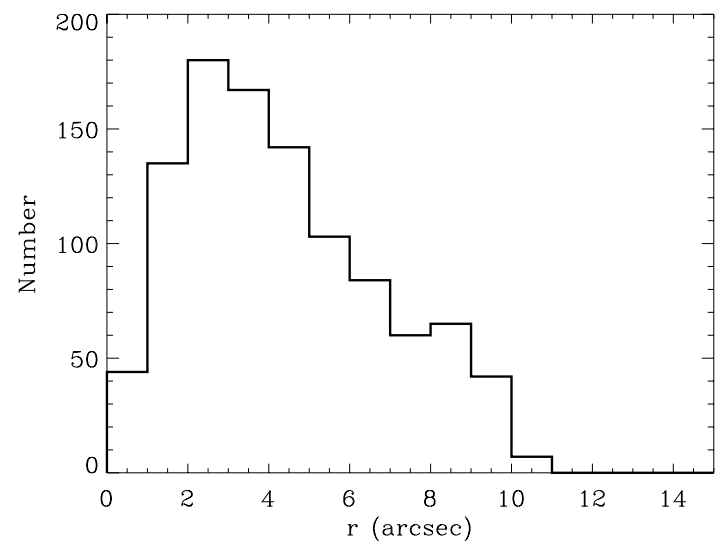

Fig. 11. FIRST - BMW-HRI: distribution of the angular separation between radio and X-ray positions for the 1019 cross-correlated objects.

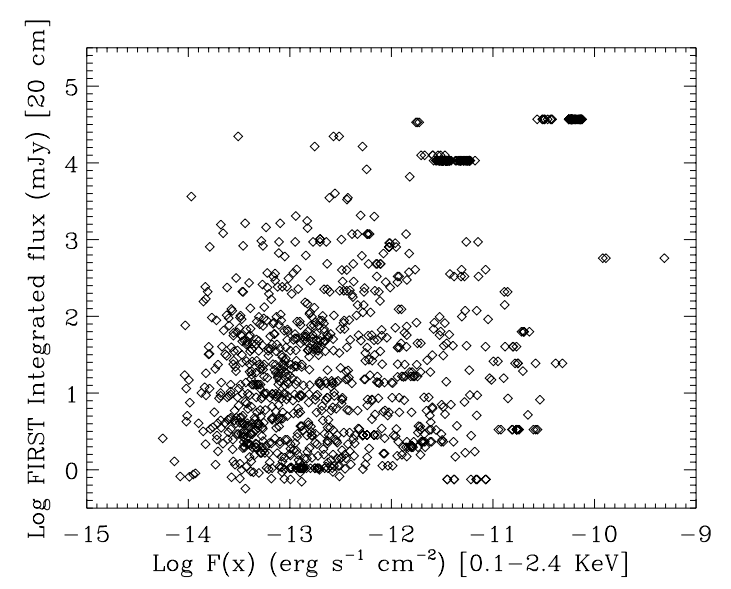

Fig. 12. FIRST - BMW-HRI: log-log plot of the integrated flux density in mJy versus X-ray flux (full column density) for the 1019 crosscorrelated objects.

plot in Fig. 13 the radio extension versus the X-ray one for the BMW-HRI objects classified as extended sources (122 entries) in the cross-correlation with FIRST. In the plot, X-ray extensions are the ones derived by our fitting procedure and subtracting in quadrature the relative PSF at a given off-axis angle. All the FIRST parameters with a brief description are reported in Appendix A (Table A.2).

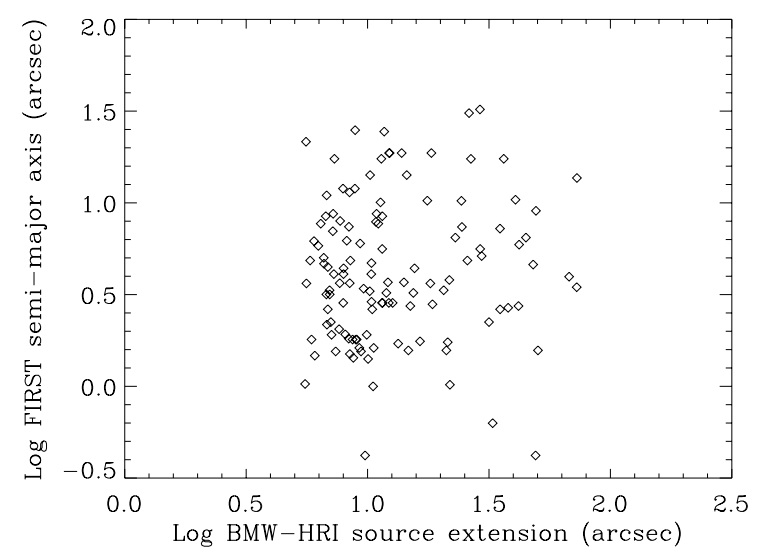

Fig. 13. FIRST - BMW-HRI: log-log plot of the major axis of the radio counterparts (that is the FWHM in arcsec of the elliptical Gaussian model) versus the X-ray extension (in arcsec) for the $\mathrm{X}$-ray sources that have been classified as extended sources (122; see the text).

\subsection{The IRAS Point Source Catalogue}

The Infrared Astronomical Satellite (IRAS) conducted a survey of $98 \%$ of the sky, from January to November 1983, in four wavelength bands centered at 12, 25, 60, and $100 \mu \mathrm{m}$ leading to the IRAS Point Source Catalogue (PSC). The catalogue contains some 250000 sources (Beichman et al. 1988). Away from confused regions of the sky, the PSC is complete to about $0.4,0.5,0.6$, and $1.0 \mathrm{Jy}$ at $12,25,60$, and $100 \mu \mathrm{m}$. The angular resolution of sources detected by IRAS varied between about $0.5 \operatorname{arcsec}$ at $12 \mu \mathrm{m}$ to about $2 \operatorname{arcmin}$ at $100 \mu \mathrm{m}$. The positional accuracy depends on source size, brightness and spectral energy distribution but is usually better than 20 arcsec. Using a cross-correlation radius of 20 arcsec (because of the IRAS positional accuracy) we found 1149 identifications with a misidentification probability of $\sim 2 \%$ (20 mismatches). We note that all the objects that have been found in common with this catalogue have been detected in all four IRAS bands. In Fig. 14 we plot the distributions of the angular separation ( $r$ in $\operatorname{arcsec})$ between the infrared and the $\mathrm{X}$-ray position for the matched objects.

Figure 15 shows for example the $12 \mu \mathrm{m}$ flux (in mJy) versus X-ray flux (full column density) for the 1149 cross-correlated sources. All the IRASPSC parameters with a brief description are reported in Appendix A (Table A.3). 


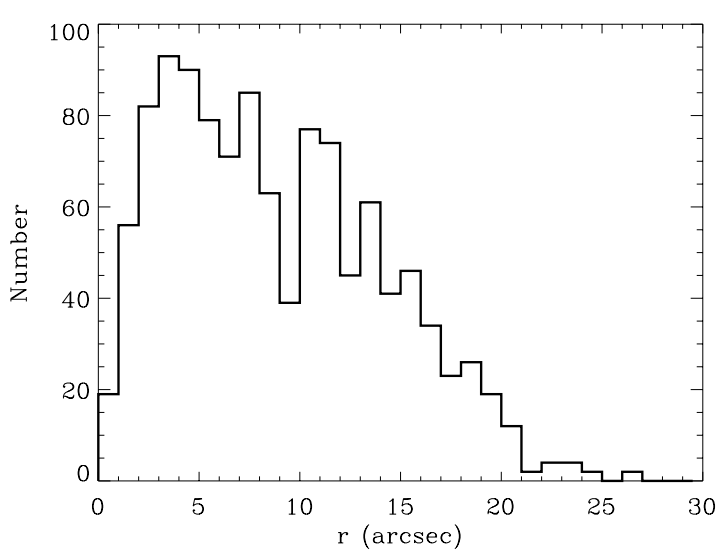

Fig. 14. IRAS - BMW-HRI: distribution of the angular separation between infrared and X-ray positions for the 1149 cross-correlated objects.

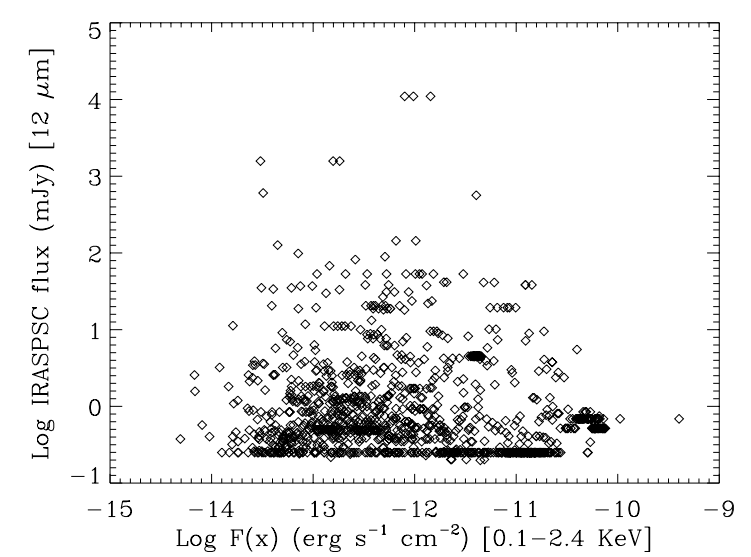

Fig. 15. IRAS - BMW-HRI: $12 \mu \mathrm{m}$ flux versus X-ray flux (full column density) for the 1149 cross-correlated objects.

\subsection{The 2MASS Survey Catalogue}

The Two Micron All Sky Survey covers over $19600 \mathrm{deg}^{2}$ $(\sim 50 \%)$ of sky observed from both the hemispheres. The catalogue contains positional and photometric information for $162,213,354$ point and 585056 extended sources observed in the three bands $J(1.25 \mu \mathrm{m}), H(1.65 \mu \mathrm{m})$ and $K_{\mathrm{s}}$ $(2.16 \mu \mathrm{m})$. The nominal survey completeness limits are 15.8 , 15.1 and 14.3 mag respectively. We have cross-correlated the BMW-HRI with the 2MASS Point Source Catalogue 2000 (Second Incremental Release) finding 7900 entries with a misidentification probability of $\sim 28 \%$ (that is 2174 mismatches). The number of $\mathrm{X}$-ray sources found to have an infrared counterpart with a measure in all the three bands is 7624 .

In Fig. 16 we report the distributions of the angular separation $(r$ in $\operatorname{arcsec})$ between the infrared and $\mathrm{X}$-ray position for the 7900 matched objects. Figure 17 shows, for example, $m_{J}$ magnitude versus X-ray flux (full column density) for the cross-correlated sources with a measured $J$ magnitude (7644). A fraction of the cross-correlated objects, the diagonal line in Fig. 17, shows a rather good correlation between X-ray and infrared fluxes probably due to common dependence on the distance (see also Fig. 19 for the cross-correlation between GSC2

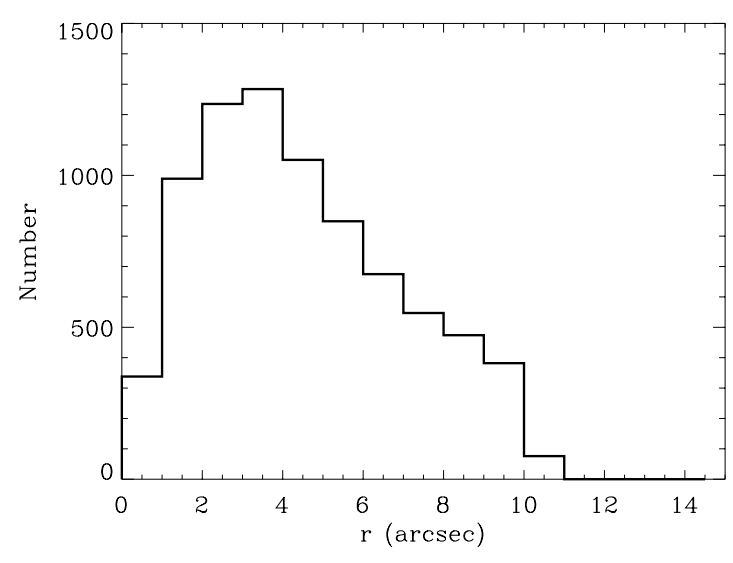

Fig. 16. 2MASS - BMW-HRI: distribution of the angular separation between infrared and X-ray positions for the 7900 cross-correlated objects.

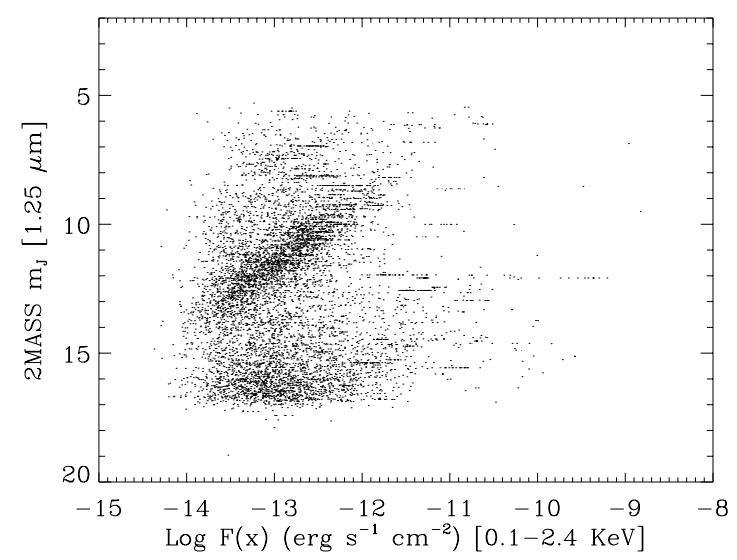

Fig. 17. 2MASS - BMW-HRI: $m_{J}$ magnitude versus logarithmic $\mathrm{X}$-ray flux (full column density) for the cross-correlated objects.

and the BMW-HRI). All the 2MASS parameters with a brief description are reported in Appendix A (Table A.4).

\subsection{The Guide Star Catalogue 2}

As a reference for our cross-correlations in the optical, we used the extended, yet unpublished, version of the recently released Guide Star Catalogue 2 (GSC2). The GSC2 is an all-sky, multi-epoch and multi-colour optical catalogue based on the digitization of $\approx 8000$ Schmidt plates obtained from 13 photographic surveys carried out between 1953 and 1991 (McLean et al. 2002, in preparation). The catalogue presently includes more than 1 billion objects. The GSC2 is aimed at providing an all-sky coverage in three photographic bands, namely $J, F$ and $N$ (roughly comparable to Johnson $B, R$ and $I$ filters). In its current version (GSC2.2), the GSC2 covers the entire sky in $J$ and $F$ bands only, while the coverage in the $N$ passband is being completed for GSC2.3. In addition, a partial coverage in the Northern hemisphere and in the South Galactic Plane is available in the $V$ band (roughly comparable to Johnson $V$ filter). The limiting magnitudes for GSC2 are $\sim 22.5-23, \sim 20-22$ and $\sim 19.5$ in the $J, F$ and $N$ bands, respectively. In the $V$ band, the limiting magnitudes are $\sim 19.5$ in the North and $\sim 14$ in the South Galactic Plane region. The photometry is accurate within 


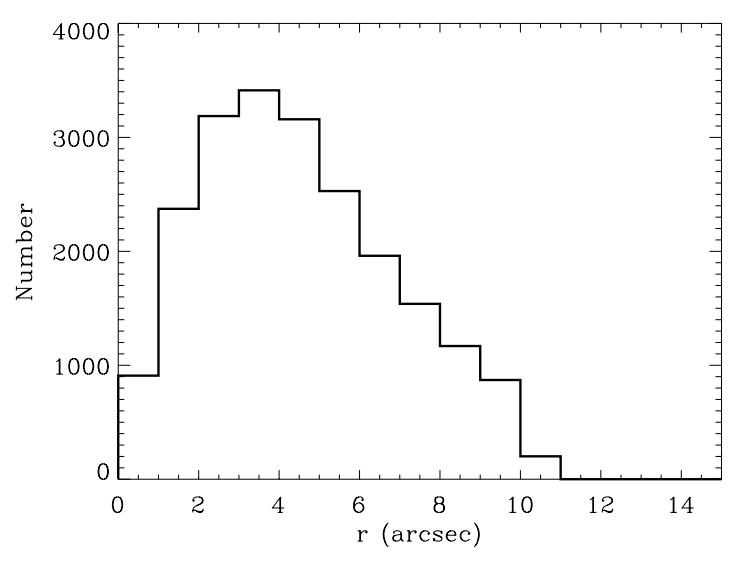

Fig. 18. GSC2 - BMW-HRI: distribution of the angular separation between optical and X-ray positions for the 21311 cross-correlated objects.

$0.2 \mathrm{mag}$ at $J=20$. The astrometry has been calibrated using as a reference the coordinates of stars extracted from the Tycho (Høg et al. 2000) and Hipparcos (Perryman et al. 1997) catalogues and have an absolute, intrinsic, accuracy of $\sim 0.3$ arcsec. The GSC2 provides also morphological classifications for all the objects detected in at least two bands, with a $\sim 90 \%$ confidence level for objects in at $|b| \geq 5^{\circ}$ and brighter than $J \sim 19$.

The cross-correlation with the BMW-HRI found 21311 entries indicating that an optical identification will be available for a sizeable fraction of BMW-HRI sources. The mismatches are 10508 , that is a misidentification probability of $\sim 50 \%$. This clearly indicates that the number of optical objects at the GSC2 limiting magnitude is very high and that for many objects one finds more than one association within 10 arcsec beam-size. In fact we found that 4822 have two possible counterparts while 2945 have more than two associations. This situation can be improved for those ROSAT HRI fields for which more than two X-ray sources can be tied to the optical reference (i.e. boresight correction). The total number of GSC2 non-star objects in the 21311 cross-correlations is 9696, while 11331 are objects classified as "star" (284 are without classification). The number of X-ray sources found to have an optical counterpart with a magnitude measure in the $J$ and $F$ bands (all-sky coverage) is 13951 , while is 68 if we also consider the $N$ band (coverage completed for GSC2.3).

A cross-correlation with the GSC (limiting magnitude about 15 ) yields only $\sim 6500$ objects. This indicates that the BMW-HRI source population has a large number of optical matching within the 15-22 mag range. In Fig. 18 we report the distributions of the angular separation $(r$ in $\operatorname{arcsec})$ between the optical and the X-ray position for the 21311 matched objects.

Figure 19 shows, for example, $m_{J}$ magnitude versus $X$-ray flux (full column density) for the cross-correlated sources with a measured $J$ magnitude (18421 objects).

Since in the GSC2 we have an information about the source extension (the semi-major axis of the fitting ellipse) we plot in Fig. 20 the optical extension versus the X-ray extension for the BMW-HRI sources that have been classified as extended sources and having an optical counterpart with a measure of the semi-major axis (1470 entries). In the plot the X-ray

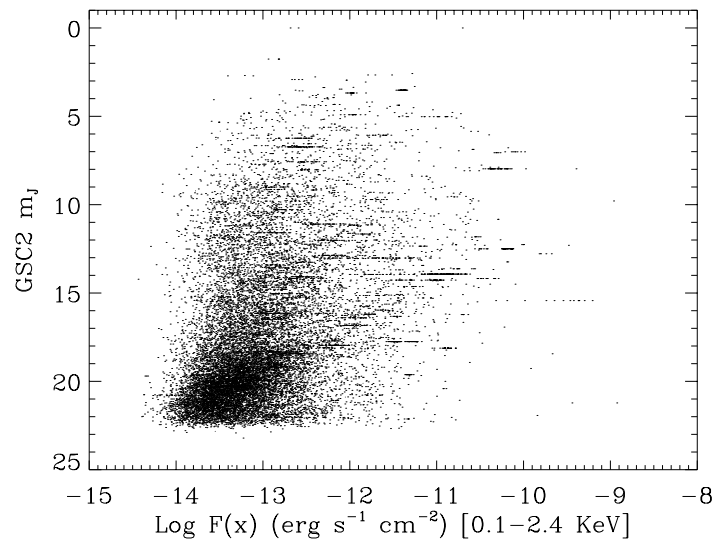

Fig. 19. GSC2 - BMW-HRI: $m_{J}$ versus logarithmic X-ray flux (full column density) for the cross-correlated objects.

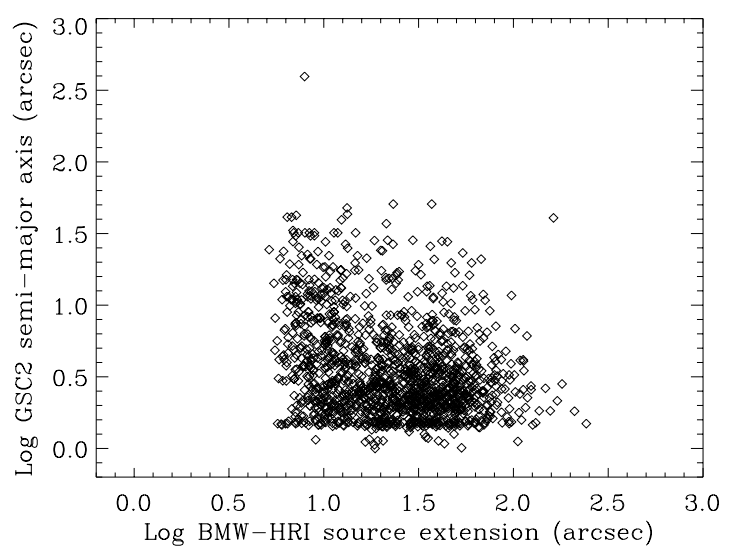

Fig. 20. GSC2 - BMW-HRI: log-log plot of the semi-major axis of the fitting ellipse (in arcsec) of the optical counterparts versus the X-ray extension (in arcsec) for the $\mathrm{X}$-ray sources that have been classified as extended sources (1470 entries; see the text).

extension is that derived from our fitting procedure subtracting in quadrature the computed PSF. We note that 1118 optical associations out of 1470 have been classified as non-star in the GSC2, 330 as star while 22 are without a classification. For the 330 optical objects flagged as a "star" in the GSC2 and with an extended X-ray counterparts we are probably in presence of blending sources or of low intensity sources at high off-axis in the X-ray images. We note that 133 of them have off-axis $\geq 10$ arcmin and that 65 of these have $S / N<5$. While the number of sources with $S / N<5$ is 107 out of 330. All the GSC2 parameters with a brief description are reported in Appendix A (Table A.5).

The number of BMW-HRI sources with an association in all the 4 catalogue (FIRST, IRASPSC, 2MASS and GSC2) is 51 . All but 6 are classified as stars in the GSC2 catalogue.

For clarity in Table 2 we report the main informations about the FIRST, IRASPSC, 2MASS and GSC2 catalogues and the results of the cross-correlations with the BMW-HRI.

\section{Summary}

The BMW-HRI catalogue counts all sources detected through a wavelet based algorithm over the entire set of available 
Table 2. Cross-correlation with other catalogues.

\begin{tabular}{lllllll}
\hline \hline Catalogues & Wavelength & $\begin{array}{l}\text { All sky fraction } \\
(\%)\end{array}$ & Entries & $\begin{array}{l}\text { Correlation } \\
\text { radius }(\operatorname{arcsec})\end{array}$ & Cross-correlations & Mismatches \\
\hline FIRST & $20 \mathrm{~cm}$ & $\sim 25$ & $\sim 770000$ & 10 & 1019 & 18 \\
\hline IRASPSC & $12-25-60-100 \mu \mathrm{m}$ & $\sim 98$ & $\sim 250000$ & 20 & 1149 & 20 \\
\hline 2MASS & $1.25-1.65-2.16 \mu \mathrm{m}$ & $\sim 50$ & 162213354 (point) & 10 & 7900 & 2174 \\
\hline GSC2 & $J-F-N-V$ & all sky $(J, F)$ & $>1$ billion objects & 10 & 21311 & 10508 \\
\hline
\end{tabular}

ROSAT HRI images with exposure time greater than $100 \mathrm{~s}$. The BMW-HRI catalogue contains 29089 sources found in 4303 fields with a detection probability of $\geq 4.2 \sigma$. For each source, the primary catalogue entries provide name, position, count rate, flux and extension along with the relative errors. The catalogue covers an area of $732 \mathrm{deg}^{2}$ down to a limiting flux of $\sim 10^{-12} \mathrm{erg} \mathrm{s}^{-1} \mathrm{~cm}^{-2}$. The detection thresholds over the entire field of view were calculated by means of simulations and extensive Monte Carlo tests. This allowed us to recover the $\log (N)-\log (S)$ distribution for cosmological sources down to a flux of $\simeq 1.2 \times 10^{-14} \mathrm{erg} \mathrm{s}^{-1} \mathrm{~cm}^{-2}$ (see Sect. 4). The BMW-HRI catalogue has been compared with both the short and long version of the ROSHRICAT catalogue and the cross-correlation for the high confidence detections is at the $\sim 90 \%$ level and at the $\sim 71 \%$ level for the long version (see Appendix A). To test the utility of the BMW-HRI catalogue in searching for possible counterparts at different wavelengths, we crosscorrelated it with some of the largest existing catalogues: the radio FIRST survey catalogue, the infrared IRAS PSC catalogue, the near-infrared 2MASS catalogue and the optical Guide Star Catalogue 2 (Sect. 6). For the radio and infrared wavelength $\sim 4 \%$ of the BMW-HRI sources have an association in the FIRST and in the IRASPSC catalogues, respectively. While $\sim 27 \%$ have a probable counterpart in the nearinfrared (2MASS catalogue). As the 2MASS survey covers $\sim 50 \%$ of sky this result is comparable with the one obtained in the optical (all-sky survey), from the cross-correlation between BMW-HRI and GSC2 ( $F$ band). If we consider all the GSC2 bands $\sim 73 \%$ of the BMW-HRI sources have an optical associations.

In order to access the service of the BMW-HRI catalogue a WEB based browser (via the DIANA ${ }^{1}$ Interface) with extensive access to multiwavelength information has been built up and can be found at: http: //www . asdc.asi.it/diana/

The source by coordinate environment allows the search by object name or coordinates and to choose the output format (table only or table and sky chart). Full catalogue information is available via the HEADS - High Energy Astrophysics Database Service on line Service (mirror of HEASARC at the Osservatorio Astronomico di Brera) with a remote telnet to ares.merate.mi.astro.it with "xray" as login (no password needed) and typed "browse bmw" to access the catalogue.

${ }^{1}$ DIANA is a joint effort of three Italian astronomical observatories (Brera, Palermo and Roma) and the Italian Space Agency's Science Data Center (ASDC). DIANA is a step towards the creation of a modern high energy astrophysics archive and advanced database system with extensive access to multiwavelength information.
Acknowledgements. We thank A. Mistò for his help with the database software. We are grateful to P. Giommi for discussions and M. Capalbi for help with the WEB based browser via the DIANA Interface. This research made use of data obtained through the NASA's HEASARC at GSFC and through the archive at MPI. This publication makes use of data products from FIRST, IRAS, 2MASS, GSC2 surveys. The 2MASS Survey is a joint project of the University of Massachusetts and the Infrared Processing and Analysis Center/California Institute of Technology, funded by the NASA and the National Science Foundation. The Guide Star Catalogue 2 is a joint project of the Space Telescope Science Institute and the Osservatorio Astronomico di Torino. STSI is operated by the Association of Universities for Research in Astronomy, for the NASA under contract NAS5-26555. The participation of the Osservatorio Astronomico di Torino is supported by the Italian Council for Research in Astronomy. Additional support is provided by ESO, ST-ECF, the International GEMINI project and the ESA Astrophysics Division. This work was supported through Cofin, CNAA and ASI grants.

\section{Appendix A: Comparison between BMW-HRI and ROSHRICAT catalogues}

\section{A.1. Cross-correlation between BMW-HRI and ROSHRICAT-short}

In the following we report all the checks done on the 1038 ROSHRICAT-short sources which lack a BMW-HRI counterpart (see Sect. 5.1). Single cases were studied as a function of their $S / N$.

(a) ROSHRICAT-short sources with $S / N \geq 20$ (20 objects). We found that all but one of the sources have a counterpart in the BMW-HRI catalogue. If we extend the cross-correlation radius we found that 18 sources have a counterpart within $\sim 20$ arcsec and one within $\sim 30$ arcsec. We checked each of these source to investigate possible mismatches. For the remaining source we checked that it is a spurious detection near a very bright source (with a positional shift of 1.5 arcmin).

(b) ROSHRICAT-short sources with $S / N$ between 5 and 20 (358 objects). To minimize the number of mismatches, we cross-correlated these sources using a radius of 18 arcsec, corresponding to the $3 \sigma$ on the combined positional error of the two catalogues. The cross-correlation found 369 BMW-HRI sources corresponding to 221 ROSHRICAT sources, i.e., 137 ROSHRICAT objects have no counterpart in our catalogue. We checked $20 \%$ of these sources (28 objects) finding that: (i) $47 \%$ are spurious detections close to bright sources; (ii) $32 \%$ are associated with extended emission and likely spurious since the SASS detection algorithm is not suited to the detection of extended sources; (iii) $7 \%$ are from a blending of two nearby point-like sources; (iv) $14 \%$ are detected with a $S / N$ lower than 
Table A.1. BMW-HRI parameters.

\begin{tabular}{|c|c|}
\hline BMW Parameter & Description \\
\hline Source Name & name of cataloged detection following IAU conventions, e.g. 1BMW143615.8+524825 \\
\hline RA & source right ascension (J2000, hhmmss.) \\
\hline DEC & source declination (J2000, ddmmss.) \\
\hline RA error & error in RA (arcsec) \\
\hline DEC error & error in DEC $(\operatorname{arcsec})$ \\
\hline Tot error & total positional error $(\operatorname{arcsec})$ \\
\hline JRA & source RA (J2000, degrees) \\
\hline JDEC & source DEC (J2000, degrees) \\
\hline LII & source Galactic Longitude (degrees) \\
\hline BII & source Galactic Latitude (degrees) \\
\hline Rebin & rebin of the subimage in which the source was found \\
\hline $\mathrm{X}$ pixel & source $x$ coordinate (pixels) \\
\hline Y pixel & source $y$ coordinate (pixels) \\
\hline Offaxis & offset from Target position (arcmin) \\
\hline Field & field name \\
\hline Target RA & target RA (J2000, hhmmss.) \\
\hline Target DEC & target DEC (J2000, ddmmss.) \\
\hline ROR & ROR number (e.g. 200005) \\
\hline Sequence & observation sequence id (e.g. rh200005a00) \\
\hline Start obs date & start observation date (ddmmyy) \\
\hline Start obs time & start observation time (hh:mm:ss.) \\
\hline End obs date & end observation date \\
\hline End obs time & end observation time \\
\hline Exposure & exposure live time (seconds) \\
\hline Map exposure & exposure from the exposure map (seconds) \\
\hline Num reb1 & number of sources detected at reb $=1$ \\
\hline Num reb3 & number of sources detected at reb $=3$ \\
\hline Num reb6 & number of sources detected at reb $=6$ \\
\hline Num reb10 & number of sources detected at reb $=10$ \\
\hline SNR & signal-to-noise ratio for detection \\
\hline Wavelet SNR & signal-to-noise ratio in the wavelet space \\
\hline Probability & probability for detection \\
\hline Count rate & estimate of net count rate $\left(\mathrm{CR}\right.$, count $\left.\mathrm{s}^{-1}\right)$ \\
\hline Fit CR error & count rate error from the fit procedure (count s $\mathrm{s}^{-1}$ ) \\
\hline Stat CR error & count rate error from statistics ( $\operatorname{count~} \mathrm{s}^{-1}$ ) \\
\hline CR error & count rate error (maximum between fit CR error and stat CR error) \\
\hline Total counts & total counts detected (CR by exposure) \\
\hline Vignetting & vignetting correction \\
\hline PSF & PSF correction \\
\hline Counted CR & source $\mathrm{CR}$ from the counting procedure \\
\hline Counted CR error & source counted CR error \\
\hline Background & background count rate \\
\hline Near sources & number of sources within 2 sigma from the detected source \\
\hline $\mathrm{Nh}$ & weighted average $\mathrm{nH}\left(\mathrm{cm}^{-2}\right)$ \\
\hline Conversion Factor 1 (CF1) & conversion factor from null column density $\left(\mathrm{erg} \mathrm{cm}^{-2}\right.$ count $\left.^{-1}\right)$ \\
\hline Fx1 & HRI X-ray flux from CF1 \\
\hline Conversion Factor1 (CF2) & conversion factor from full column density $\left(\mathrm{erg} \mathrm{cm}^{-2} \mathrm{count}^{-1}\right.$ ) \\
\hline Fx2 & HRI X-ray flux from CF2 \\
\hline Extension & sigma source in original pixels $(\operatorname{arcsec})$ \\
\hline Extension error & sigma source error \\
\hline Flag sigma & fixed sigma? $(\mathrm{Y} / \mathrm{N})$ \\
\hline Flag extended & extended sources? (POINT/EXTENDED) \\
\hline Extension significativity & significativity of the extension \\
\hline Fit $\chi^{2}$ & $\chi^{2}$ from the fit procedure \\
\hline Cat version & catalogue version \\
\hline SASS version & version of the SASS processing system \\
\hline
\end{tabular}


Table A.2. FIRST parameters.

\begin{tabular}{ll}
\hline \hline FIRST Parameter & Description \\
\hline FIRST name & FIRST source name (e.g. 11030+38071E) \\
RA & FIRST source RA (J2000, degrees) \\
DEC & FIRST source DEC (J2000, degrees) \\
W & warning flag for sidelobe source \\
Pflux & peak flux density (mJy) \\
Iflux & integrated flux density (mJy) \\
Rms & local noise at the source position (mJy) \\
Maj & major axis after deconvolution $(F W H M$ in arcsec; elliptical Gaussian model) \\
Min & minor axis after deconvolution (in arcsec) \\
PA & position angle after deconvolution (in arcsec; degrees east of north) \\
fMaj & measured major axis (arcsec) \\
fMin & measured minor axis (arcsec) \\
fPA & measured position angle (arcsec) \\
FIRSTBMW & angular distance between FIRST and X-ray position (arcsec) \\
FIRST comp & number of FIRST cross-correlations \\
BMWFIRST version & BMW-FIRST cross-correlation version \\
\hline
\end{tabular}

Table A.3. IRASPSC parameters.

\begin{tabular}{ll}
\hline \hline IRASPSC Parameter & Description \\
\hline IRASPSC name & source IRASPSC name (e.g. 18352+3844) \\
RA & IRASPSC RA source (J2000, degrees) \\
DEC & IRASPSC DEC source (J2000, degrees) \\
F12 & 12 micron flux (mJy) \\
F25 & 25 micron flux (mJy) \\
F60 & 60 micron flux (mJy) \\
F100 & 100 micron flux (mJy) \\
IRASPSCBMW & angular distance between IRAS and X-ray position $(\operatorname{arcsec})$ \\
IRASPSC comp & number of IRASPSC cross-correlations \\
IRASPSC version & BMW-IRASPSC cross-correlation version \\
\hline
\end{tabular}

Table A.4. 2MASS parameters.

\begin{tabular}{ll}
\hline \hline 2MASS Parameter & Description \\
\hline RA & 2MASS RA source (J2000, degrees) \\
DEC & 2MASS DEC source (J2000, degrees) \\
$J$ mag & 2MASS $J$ magnitude \\
$J$ mag error & 2MASS $J$ magnitude error \\
$H$ mag & 2MASS $H$ magnitude \\
$H$ mag error & 2MASS $H$ magnitude error \\
$K$ mag & 2MASS $K$ magnitude \\
$K$ mag error & 2MASS $K$ magnitude error \\
2MASSBMW & angular distance between 2MASS and X-ray position (arcsec) \\
2MASS comp & number of 2MASS cross-correlations \\
BMW2MASS version & BMW-2MASS cross-correlation version \\
\hline
\end{tabular}

what reported by the SASS and we recover them if we use a lower detection threshold (detection probability of $3.5 \sigma$ instead of $4.2 \sigma)$.

(c) ROSHRICAT-short sources with $S / N<5$ (660 objects). These sources are rather faint and 16 have $S / N$ lower than 4 even in the ROSHRICAT-short itself. Their detection depends critically on the extraction radius and on local background. The great majority should have been missed by our detection algorithm because they are indeed too faint to satisfy our detection threshold (which is not based on $S / N$ ratio).

\section{A.2. Check on the 331 obvious sources not detected by the SASS and manually added}

We found that 79 of these sources are in fields we discarded (ROR number 1 and 5 or fields rejected during the analysis). 
Table A.5. GSC2 parameters.

\begin{tabular}{|c|c|}
\hline GSC2 Parameter & Description \\
\hline GSC2 name & GSC2 source name (e.g. N2320203180) \\
\hline RA & GSC2 source RA (J2000, degrees) \\
\hline DEC & GSC2 source DEC (J2000, degrees) \\
\hline RA error & source RA error (degrees) \\
\hline DEC error & source DEC error (degrees) \\
\hline GSC2 epoch & GSC2 epoch (e.g. 1991.329956) \\
\hline $\mathrm{raPM}$ & RA proper motion $\left(\right.$ mas $\left.\mathrm{yr}^{-1}\right)$ \\
\hline decPM & DEC proper motion $\left(\right.$ mas $\left.\mathrm{yr}^{-1}\right)$ \\
\hline raPMerr & RA proper motion error $\left({\left.\operatorname{mas~} \mathrm{yr}^{-1}\right)}^{-1}\right.$ \\
\hline decPMerr & DEC proper motion error $\left(\right.$ mas $\left._{\mathrm{yr}^{-1}}\right)$ \\
\hline$F$ mag & $F$ magnitude \\
\hline$F$ mag error & $F$ magnitude error \\
\hline$J$ mag & $J$ magnitude \\
\hline$J$ mag error & $J$ magnitude error \\
\hline$V$ mag & $V$ magnitude \\
\hline$V$ mag error & $V$ magnitude error \\
\hline$N$ mag & $N$ magnitude \\
\hline$N$ mag error & $N$ magnitude error \\
\hline$a$ & semi-major axis \\
\hline$e$ & eccentricity \\
\hline PA & position angle (arcsec) \\
\hline$c$ & class code $(0=$ star, $1=$ galaxy, $2=$ blend, $3=$ non-star, $4=$ unclassified, $5=$ defect $)$ \\
\hline GSC2BMW & angular distance between GSC2 and X-ray position ( $\operatorname{arcsec})$ \\
\hline GSC2 comp & number of GSC2 cross-correlations \\
\hline BMWGSC2 version & BMW-GSC2 cross-correlation version \\
\hline
\end{tabular}

We found that 231 of the 252 remaining sources have a counterpart in our catalogue: 230 within 30 arcsec and 1 within 50 arcsec. We checked with a visual inspection the remaining 21 obvious sources finding that: 8 are spurious detections; 8 are very faint sources which we are able to detect if we use a lower detection threshold for our algorithm (significance $\geq 3.5 \sigma)$; 3 sources are ambiguous since XIMAGE detects only a $3 \sigma$ upper limit; one source is at the edge and can be detected only by integrating its flux over all the HRI energy channels (see Sect. 2); the remaining one is very close to an hot spot and was classified as spurious. For the 8 spurious we note that 5 of them are not far away from bright sources. It could be that the manual insertion of the coordinates were wrong.

\section{A.3. Cross-correlation between BMW-HRI and ROSHRICAT-long}

There are 7107 BMW-HRI sources without a counterpart in the ROSHRICAT-long (see Sect. 5.2). Using a cross-correlation radius of 18 arcsec (i.e. corresponding to $3 \sigma$ on the combined average positional error of the two catalogues) the number of unmatched sources reduces to 5870 . For the visual inspection we excluded sources in fields with large extended emission and too crowded and we selected a sample consisting of 1170 sources with $S / N \geq 5$. These sources were considered and studied as a function of their $S / N$.

(a) BMW-HRI sources with $S / N \geq 20$ (27 objects): (i) $63 \%$ are bright or relatively bright objects (13 at the detector edge and 4 are targets); (ii) 26\% all with off-axis $>17$ arcmin have a ROSHRICAT counterpart with an angular separation larger than 18 arcsec; (iii) the remaining $11 \%$ are at the edge of the detector (off-axis $\geq 18$ arcmin) and are hardly recognizable at a visual inspection.

(b) BMW-HRI sources with S/N between 5 and 20 (1143objects): we checked $10 \%$ of these sources. We note that $86 \%$ of these have an off-axis angle $\geq 14$ arcmin and $40 \%$ are extended sources. We found that: (i) $36 \%$ have a counterpart in the ROSHRICAT-long with an angular separation $>18$ arcsec. The combination of extended emission with high off-axis angle can explain the highest angular separation. In fact almost all sources with angular separation $>30$ arcsec have off-axis $\geq 14$ arcmin and are extended sources; (ii) $48 \%$ are sources without a counterpart in the ROSHRICAT-long and 50\% of these are well visible at a visual inspection. We note that $85 \%$ have off-axis $\geq 14$ arcmin and that $42 \%$ are extended sources; (iii) $16 \%$ are sources of ambiguous interpretation: all these sources but one have off-axis $\geq 17$ arcmin and seem fluctuations of the background at a visual inspection. We note that $11 \%$ of these are extended sources. If we use the XIMAGE 
package at the positions of the point sources (16 objects) we find a $3 \sigma$ upper limits for 11 sources while for the remaining 5 the algorithm detects sources with very low signal to noise ratio: 4 out 5 with $S / N \leq 2$ and the remaining with $S / N \sim 6$.

\section{References}

Becker, R. H., White, R. L., \& Helfand, D. J. 1995, ApJ, 450, 559

Beichman, C. A., et al. 1988, Infrared Astronomical Satellite (IRAS) Catalogs and Atlases, vol. 1, Explanatory Supplement, NASA RP-1190 (Washington, DC: GPO)

Campana, S., Lazzati, D., Panzera, M. R., \& Tagliaferri, G. 1999, ApJ, 524,423

David, L. P., et al. 1998, The ROSAT HRI Calibration Report, U.S. ROSAT Science Data Center (SAO)

Dickey, J. M., \& Lockman, F. J. 1990, ARA\&A, 28, 215

Gioia, I. M., Maccacaro, T., Schild, R. E., et al. 1990, ApJS, 72, 567

Giommi, P., Tagliaferri, G., Beuermann, K., et al. 1991, ApJ, 378, 77

Gotthelf, E. V., \& White, N. E. 1997, in International Symposium on X-ray Astronomy ASCA Third Anniversary, ed. F. Makino, \& K. Mitsuda, 31

Guzzo, L., et al. 2002, in preparation

Hasinger, G., et al. 1998, A\&A, 329, 482

Høg, E., Fabricius, C., Makarov, V. V., et al. 2000, A\&A, 357, 367
Lazzati, D., Campana, S., Rosati, P., et al. 1998, A\&A, 331, 41

Lazzati, D., Campana, S., Rosati, P., et al. 1999, ApJ, 524, 414

McLean, B., et al. 2002, in preparation

Moretti, A., et al. 2002, in preparation

Pavlov, G. G., Zavlin, V. E., Aschenbach, B., et al. 2000, ApJ, L53

Perryman, M. A. C., Lindegren, L., Kovalevsky, J., et al. 1997, A\&A, 323, L49

Pfeffermann, E., et al. 1986, Proc. SPIE, 773, 519

Prestwich, A., Callanan, P., Snowden, S., et al. 1996, AAS, 189, 905

ROSAT Scientific Team 2001, ROSAT NEWS No. 74

Rosati, P., Della Ceca, R., Burg, R., et al. 1995, ApJ, 445, L11

Snowden, S. L. 1994, Cookbook for analysis procedures for ROSAT XRT/PSPC observations of extended objects and diffuse background

Trümper, J., et al. 1991, Nature, 349, 579

Voges, W., Aschenbach, B., Boller, Th., et al. 1999, A\&A, 349, 389

White, N. E., Giommi, P., \& Angelini, L. 1994, IAU Circ., 6100

White, R. L., Becker, R. H., Helfand, D. J., \& Gregg, M. D. 1997, AJ, 475,479

Zimmermann, H. U. 1994, IAU Circ., 6102

Zombeck, M. V., Conroy, M., Harnden, F. R., et al. 1990, in Proc. SPIE Conf. EUV, X-Ray, and Gamma-ray Instrumentation for Astronomy, Calibration of the ROSAT High Resolution Imager, 1344, 267 